\title{
Development and evaluation of a service-learning model for preclinical student education in cardiovascular disease prevention
}

This article was published in the following Dove Press journal:

Advances in Medical Education and Practice

II March 2016

Number of times this article has been viewed

\author{
Nilay S Shah* \\ Jasmine Rassiwala* \\ Allison L Ducharme-Smith \\ David A Klein \\ Ashley S Kim \\ Claudia Leung \\ Rabih Dahdouh \\ Stephen Havas
}

Department of Preventive Medicine, Northwestern University Feinberg

School of Medicine, Chicago, IL, USA

*These authors contributed equally to this work
Correspondence: Nilay S Shah

Department of Preventive Medicine, Northwestern University Feinberg School of Medicine, 680 N. Lake Shore Drive, Suite I400, Chicago, IL 606 II, USA Email nilay-shah@fsm.northwestern.edu
Background: Cardiovascular diseases are the leading cause of preventable morbidity and mortality in the USA. Medical schools must prepare trainees to address prevention, including improving ability in counseling patients to modify lifestyle risk factors. Most medical students do not receive significant training or clinical experience in preventive medicine until the clinical years of medical school. To enhance student education in disease prevention and lifestyle counseling, and simultaneously target cardiovascular disease prevention in high-risk Chicago neighborhoods, the Northwestern University Feinberg School of Medicine and Chicago Department of Public Health with support from the GE Foundation, developed the Keep Your Heart Healthy program.

Methods: Medical students participated in intensive faculty-led training. They subsequently screened local residents to identify and counsel for cardiovascular disease risk factors. Fifty-one predominantly preclinical medical students screened residents of the Humboldt Park and North Lawndale neighborhoods in Chicago, IL, at 31 screening events from August to December 2013. Fifty students ( $98 \%$ response rate) completed a survey assessing the educational value of various program components following the pilot.

Results: Of all respondents, $92 \%$ of students reported improved knowledge of cardiovascular disease prevention and $94 \%$ reported improved knowledge of vulnerable populations and health equity. The majority ( $88 \%$ ) reported that their participation supplemented material they learned in the classroom. Eighty-six percent of students reported that their encounters with community participants were of educational value. Integration of this program into the medical school curriculum was supported by $68 \%$ of students.

Conclusion: Keep Your Heart Healthy educates primarily preclinical medical students in cardiovascular disease prevention and prepares them to apply this knowledge for patient counseling. Results from student surveys demonstrate that this service-learning initiative enhances medical student knowledge in cardiovascular disease prevention, supplements classroom material, and provides students a valuable opportunity to apply interviewing and counseling skills in a real patient encounter.

Keywords: service learning, undergraduate medical education, cardiovascular disease, prevention, community health

\section{Background}

Cardiovascular diseases (CVDs) remain the leading cause of morbidity and mortality in the USA. ${ }^{1}$ CVD is largely preventable with lifestyle modification and targeted drug treatments. The estimated annual direct and indirect costs of CVD were \$313 billion in 2009. ${ }^{2}$ In 2011, the Chicago Department of Public Health (CDPH) launched its Healthy Chicago public health agenda in order to improve the overall physical and mental 
health of its residents. ${ }^{3}$ Healthy Chicago priorities that target CVD include heart disease and stroke prevention and obesity prevention. The Keep Your Heart Healthy (KYHH) program was developed as a partnership between CDPH, Northwestern University Feinberg School of Medicine (NUFSM), and community partners to address these citywide goals.

At the community level, KYHH aims to screen at-risk Chicago residents for CVD risk factors, counsel them on behavior and risk factor modification, and refer participants to primary care follow-up. Results from previous programs suggest that medical students' involvement can benefit low-resource communities through health counseling and education. ${ }^{4}$ This evidence indicates that medical students serve as strong advocates for patient health, which can lead to improved outcomes.

Beyond targeting community health improvement, $\mathrm{KYHH}$ is also an educational initiative for medical students. Student involvement in community-based programs is a successful method of experiential service-learning for education. ${ }^{5,6}$ Results from prior programs indicate that students participating in community volunteer work learn to interact with people of different backgrounds and improve communication skills. Students also develop new clinical skills such as taking blood pressure measurements, and gain a greater understanding of issues affecting local neighborhoods. ${ }^{5,7}$ Many reports have detailed the role of community health workers $(\mathrm{CHWs})^{8,9}$ and lay health workers ${ }^{10,11}$ in community-based cardiovascular (CV) risk reduction programs. However, the available evidence for medical students is largely limited to third- and fourth-year students on their clinical rotations at community hospitals and in free clinics. ${ }^{12-15}$ Data are sparse regarding community-based intervention programs involving preclinical medical students outside of free clinics.

Traditionally, medical students participate in servicelearning informally, such as in student-run clinics or health fairs. KYHH is unique as it is a more formal, structured community-based service-learning program that involves predominantly preclinical students in the screening and counseling for CVD risk. Participation in KYHH provides students with an opportunity to apply medical knowledge and skills in nonclinical settings. We hypothesized that student participation in $\mathrm{KYHH}$ would increase student knowledge of disease prevention and improve student comfort and ability in counseling and interpersonal communication. Overall, the didactic training and applied experience in which medical students participate is intended to align with NUFSM educational competencies. These competencies are based on Accreditation Council for Graduate Medical Education (ACGME) objectives for medical education. Here we detail the development, pilot implementation, and initial evaluation of KYHH as a servicelearning model for medical student education at NUFSM.

\section{Methods}

\section{Program development}

In 2012, KYHH was founded through collaboration between NUFSM, CDPH, and the GE Foundation. The NUFSM principal investigator recruited eight university faculty members to serve on a KYHH Executive Committee. The Executive Committee included the Chair of the Department of Preventive Medicine, the Director of the NUFSM Institute for Public Health and Medicine, the NUFSM Vice Dean for Education, the Commissioner of CDPH, a medical student representative, and faculty experts on nutrition, motivational interviewing, community health, and CVD prevention. The Executive Committee advised the NUFSM principal investigator on project development and led medical student training sessions.

A Student Advisory Committee (SAC) was created, comprising two third-year medical student chairs, three additional third-year medical students, two second-year students, and four first-year medical students. During the development of KYHH, a senior research study coordinator was hired to organize project communication and activities. Together, the Executive Committee and SAC identified guiding objectives of KYHH, which included: 1) aligning with and strengthening the NUFSM medical student educational curriculum's core competencies; 2) addressing a critical community and public health priority; and 3) completing a valid evaluation of the project.

The Chicago communities of Humboldt Park and North Lawndale were selected as pilot neighborhoods since they have been previously identified as under-resourced, lowincome communities with large populations of two high-risk racial minority groups (Hispanic and African-American, respectively). KYHH partnered with the Greater Humboldt Park Community Diabetes Empowerment Center and Family Focus Lawndale organizations in each respective neighborhood. Each community organization is linked with a local Federally Qualified Health Center (FQHC). CHWs from each of these organizations recruited local participants for screening. The multidisciplinary Executive Committee, which included experts in creating surveys for nutrition and physical activity, developed a screening questionnaire for students to administer at screening events (see Figure S1). A Spanish translation was created to ensure cultural sensitivity and appropriateness of the content. The Institutional Review Board at NUFSM, which reviews and approves all research 
projects, determined that KYHH qualified for an exemption as a research and demonstration project for public health.

\section{Faculty and student recruitment}

Prior to KYHH screening events, the study coordinator recruited NUFSM attending physicians and an Internal Medicine chief resident to supervise medical students at screening events. The SAC sent a brief survey to the NUFSM student body gauging interest for participation in KYHH. Based on positive feedback, the SAC recruited a pilot group of students to participate in the community screening events. Students were recruited via email participation surveys, interest meetings, and announcements during classes. Prior to participation, students were required to attend two, 2-hour long training sessions led by faculty experts.

\section{Training session content}

Training sessions consisted of a combination of formal didactic teaching and interactive counseling practice. Didactic lectures covered the epidemiology and prevention of CVD, the American Heart Association's Life's Simple 7 recommendations, ${ }^{16}$ and optimal dietary modification strategies for CVD risk reduction. Interactive sessions were dedicated to motivational interviewing and counseling training in simulated patient encounters. Finally, one session was devoted to training medical students about their specific role during each screening event, including how to measure blood pressure accurately and elicit pertinent clinical and CV medical history (see Figure S1 for specific questions asked).

Students were taught to refer participants for primary care follow-up for elevated blood pressures of $\geq 140 \mathrm{mmHg}$ systolic blood pressure and/or $90 \mathrm{mmHg}$ diastolic blood pressure. In the presence of CVD warning symptoms (eg, chest pain, shortness of breath, syncope), students were trained to consult the supervising attending physician to determine the need for emergent hospital referral. Students were also advised to consult the attending physician in the event of any uncertainties in the participant encounter. At the conclusion of each training session, students were asked to complete a feedback survey evaluating each lecture for content relevance. Students were also asked to provide written feedback with suggestions to improve training sessions.

\section{Screening event schedule and organization}

Screening events were held weekly in both Humboldt Park and North Lawndale. A total of 1,161 people (877 in Humboldt Park and 284 in North Lawndale) were screened during the pilot. CHWs recruited participants for screening via handouts and poster advertisements throughout the week during various community health programs. Participants were made aware that screening sessions were hosted by NUFSM and medical students were conducting the screenings under the supervision of attending physicians. Further details on results from the community screenings have been previously published. ${ }^{17}$ Additional screening events were organized for special neighborhood events (eg, festivals and food drives) at each community center. Screening and education methods were consistent for both communities. Each session was staffed by at least one supervising attending physician, the program coordinator, two to three $\mathrm{CHWs}$, and up to ten students. Students with Spanish-speaking ability were encouraged to participate at the Humboldt Park events. As needed, CHWs served as translators.

\section{Screening session content and student role}

Each screening encounter began with measurement of participant height, weight, and calculation of body mass index. Each student then administered the screening questionnaire. Students sequentially asked participants each question and recorded answers on the questionnaire. Following this, students performed blood pressure measurements. Automated blood pressure cuffs (Omron BP760; Omron Corp., Kyoto, Japan) were used for this assessment, with the participant seated in a chair with both feet on the floor, the back and arm supported, for 5 minutes prior to the first measurement. Students obtained a total of three blood pressure readings, recorded each result on the questionnaire, and recorded the average of the final two readings.

Attending physicians circulated around the studentparticipant pairs to ensure that student counseling and risk assessment were appropriate and accurate. The attending physicians offered feedback and teaching to students when necessary, for instance looking up appropriate management guidelines to direct further care. In situations when participants reported active or recent symptoms to students or had other concerns, attending physicians joined the conversation to help determine the appropriate course of follow-up for the participant. The attending physician later taught the student about the clinical relevance and significance of the participant's presentation.

Students were also encouraged to seek the input of the attending physician in any circumstance in which the student was unsure of further management. Participants with significantly elevated blood pressure (defined as systolic blood 
pressure $\geq 180 \mathrm{mmHg}$ or diastolic blood pressure $\geq 120 \mathrm{mmHg}$ ) or symptomatic participants at the attending physician's discretion, were urged to go immediately to a local emergency department. Asymptomatic participants with hypertension below these blood pressure levels were referred either to a $\mathrm{FQHC}$ or encouraged to follow-up with their primary care physician. In this case, a referral form was completed and sent to either the $\mathrm{FQHC}$ or the established primary care clinic.

Following blood pressure measurement, students prompted the participant to select a health behavior topic for discussion and counseling (see Figure S1). Students provided personalized counseling based on the health behavior topic preferences selected by community participants. These discussions focused on strategies participants could utilize to modify high-risk behaviors in order to reduce their personal CVD risk. Most of these counseling points were derived from the American Heart Association's Life's Simple 7 campaign and tailored to the cultural nuances and available local resources within the community.

After participating in at least one screening event, students were asked to complete an evaluation survey developed by the SAC. This survey was piloted to elicit students' opinions on the educational value of KYHH (see Table 1).

\section{Results}

A total of 51 students (37 first-year, four second year, two third-year, and eight fourth-year medical students) completed the two, 2-hour KYHH training sessions. The students staffed 31 screening events with each event staffed by a mean (standard deviation [SD]) of 6.8 (2.4) students. Students volunteered on average (SD) once per 7.9 (5.9) events (see Table 2 for further details).

All 51 students provided feedback after completing the training sessions. Seventy-one percent of participating students identified motivational interviewing as a strength of the training. CVD background data (59\%) and dietary guidelines for CV health (47\%) were also identified as strengths. Students indicated several areas for improvement including more practice for clinical scenarios with coaching tips (53\%) and more time for role-play of communication skills (35\%).

Fifty students, representing $98 \%$ of the students trained to participate, completed an evaluation of the educational value of $\mathrm{KYHH}$ after attending at least one screening event (see Table 1). Ninety-two percent of respondents said their participation strengthened their knowledge base in CVD prevention. Ninety-four percent of students indicated that their participation improved their education regarding vulnerable populations and health equity.
Table I Survey results from KYHH pilot

\begin{tabular}{lll}
\hline Question and response choices & N & $\%$ \\
\hline I. What is your current year in school? & & \\
MI (first-year, preclinical) & 36 & 72 \\
M2 (second-year, preclinical) & 4 & 8 \\
M3 (third-year, clinical) & 2 & 4 \\
M4 (fourth-year, clinical) & 8 & 16
\end{tabular}

2. How has your participation in $\mathrm{KYHH}$ affected your knowledge base in cardiovascular disease prevention?

Weakened my knowledge a lot $\quad 0 \quad 0$

Weakened my knowledge a little $\quad 0 \quad 0$

Had no effect on my knowledge $\quad 4 \quad 8$

Strengthened my knowledge a little $\quad 24 \quad 48$

$\begin{array}{lll}\text { Strengthened my knowledge a lot } & 22 & 44\end{array}$

3. How has your participation in $\mathrm{KYHH}$ affected your education

regarding vulnerable populations and health equity?

Did not improve my education on this topic $\quad 3 \quad 6$

$\begin{array}{lll}\text { Improved my education on this topic a little } & 23 & 46\end{array}$

Improved my education on this topic a lot $\quad 24 \quad 48$

4. Was the opportunity to apply what you have learned in the classroom and during $\mathrm{KYHH}$ trainings of educational value? Not at all educational $\quad 0 \quad 0$

Somewhat educational $\quad 28 \quad 56$

Very educational $\quad 22 \quad 44$

5. Rate the degree to which your participation in $\mathrm{KYHH}$ added to the material you have learned in the classroom?

Detracted a significant amount from my NUFSM education 00

Detracted a little from my NUFSM education $\quad 0 \quad 0$

$\begin{array}{llll}\text { Did not detract from or add to my NUFSM education } & 5 & 10\end{array}$

Added a little to my NUFSM education $\quad 28 \quad 56$

Added a significant amount to my NUFSM education $\quad 16 \quad 32$

6. Which of the following $\mathrm{KYHH}$ activities did you find to be of educational value? (Mark all that apply)

Didactic training (CVD epidemiology, nutrition lectures, etc) $\quad 28 \quad 56$

Applied training (eg, measuring BP, motivational interviewing) $\quad 32 \quad 64$

Contact/discussion with attending physicians $\quad 34 \quad 68$

Encounters with community participants at screening events $\quad 43 \quad 86$

None of these

0

7. Would you support integrating $\mathrm{KYHH}$ into the curriculum as an activity for all medical students?

Strongly against

Somewhat against

Indifferent

Somewhat for

Strongly for

24

$\begin{array}{ll}7 & 14\end{array}$

$\begin{array}{ll}6 & 12\end{array}$

$16 \quad 32$

$18 \quad 36$

Notes: Questions 5 and 7 reflect responses from all participants who answered the question. The missing data indicate that participants chose not to respond. (\%) Represents the percent of total respondents, $(\mathrm{N})$ represents the number of survey respondents providing this answer.

Abbreviations: BP, blood pressure; CVD, cardiovascular disease; KYHH, Keep Your Heart Healthy; NUFSM, Northwestern University Feinberg School of Medicine.

All survey respondents (100\%) reported that the opportunity to apply what they had learned in the classroom and during KYHH trainings was of educational value. Eightyeight percent of students reported that their participation in $\mathrm{KYHH}$ added to the material they learned through the NUFSM curriculum. Students reported that their encounters with community participants during screening events 
Table 2 Number of student participants and frequency of participation in KYHH pilot

\begin{tabular}{llllll}
\hline & First year & Second year & Third year & Fourth year & Total \\
\hline Total students on KYHH roster & 37 & 4 & 2 & 8 & 5 I \\
Number of students staffing $\geq$ I event(s) & 37 & 4 & 2 & 8 & 5 I \\
Number of students staffing $\geq 2$ events & $3 \mathrm{I}$ & 4 & 2 & 8 & 45 \\
Number of students staffing $\geq 3$ events & 23 & 4 & 2 & 8 & 37 \\
Number of students staffing $\geq 4$ events & 13 & 4 & I & 8 & 26 \\
Number of students staffing $\geq 5$ events & 5 & 4 & 0 & 17 \\
\hline
\end{tabular}

Abbreviation: $\mathrm{KYHH}$, Keep Your Heart Healthy.

were one of the most educational components of KYHH. Sixty-eight percent of students favored integration of KYHH into the NUFSM curriculum.

Individual student reflections on KYHH were overall positive (see Table 3). These testimonials highlighted the unique opportunity to work in local communities, engage one-on-one with community residents, and apply inclassroom concepts in practical settings. Student comments also reflected support for integrating KYHH into the existing NUFSM curriculum.

\section{Discussion}

KYHH is a unique initiative that enables preclinical students to gain knowledge of CVD prevention, apply medical knowledge in the community setting, build interpersonal communication skills, and serve as patient educators. As shown in this report, students felt this program enriched their medical education experience. Students reported that KYHH improved their knowledge of CVD prevention, and enhanced their education surrounding vulnerable groups and health equity. The majority felt that KYHH added to the material they learned in the classroom. The majority would also support integration of KYHH into the NUFSM curriculum. Most students also felt that their encounters with community residents were of educational value. Individual student comments noted the value of practicing motivational interviewing skills in a "real patient" setting. The program also exemplifies a successful collaboration and partnership between multiple stakeholders - namely, an academic institution, public health department, and community organizations and leaders - to develop a program of mutual benefit.

\section{Advantages of student involvement in the community}

Students' participation in community settings is increasingly recognized as an important part of medical education. The Liaison Committee on Medical Education, the primary accrediting body for schools of medicine in the USA and Canada, recommends that medical schools create sufficient opportunity for students to participate in service-learning in order to provide context to their academic coursework and emphasize future physicians' roles as citizens and professionals. ${ }^{18}$ There are limited objective outcome data which show that service learning makes a positive impact on medical student education. Prior evidence suggests that greater involvement in service-learning as a medical student may be related to above-average class rank and election to the Alpha Omega Alpha medical honor society, ${ }^{6}$ although the link is not necessarily causal.

Subjective student accounts favor service-learning as an effective educational opportunity within health professions education. ${ }^{19-22}$ These analyses suggest that increased medical student community engagement and reciprocal knowledge transfer between students, faculty, and CHWs can help prepare medical students to practice in a health care environment that increasingly places importance on accountability to the surrounding community's health. ${ }^{23}$ Prior programs suggest that community engagement experiences support health professions student understanding of social responsibility in their future careers. ${ }^{24}$ Medical students have previously indicated that engagement in service-learning supports the development of patient education skills, enhances their ability to articulate the role of physicians in the community, and promotes an understanding of how socioeconomic factors influence health care access and compliance. ${ }^{5,25,26}$

Our results build on these findings. As a nonclinic-based service-learning opportunity, KYHH provides a venue to apply skills learned in didactic and interactive training in a community setting. This community-based, rather than classroom-based or clinically based, program enhanced students' medical knowledge and understanding of vulnerable populations. Accordingly, students noted that their experience through KYHH supplemented the material they learned in the NUFSM curriculum.

\section{$\mathrm{KYHH}$ and the NUFSM competencies}

NUFSM employs a competency-based education framework for its medical students. These competencies are used to guide curricular development, define the expectations of student 
Table 3 Student participants' qualitative feedback for $\mathrm{KYHH}$

\section{Students' responses to $\mathrm{KYHH}$ participation}

"I felt like I was making a difference."

"You get to experience a lot of different things - understanding barriers to health care and wellness, motivational interviewing, and counseling. There are not too many other experiences that give you that unique patient perspective and how it all relates to the things we're learning in our curriculum."

"This program has allowed me to hone my motivational interviewing skills and to interact with a wide array of patients. It also greatly improved the confidence I have talking with patients, which I believe manifested itself in my OSCE grade."

"KYHH gives us the chance to use our time to make an impact in the community while exposing us to diversity we might otherwise not see."

"I noticed that there was significant overlap between $\mathrm{KYHH}$ and what we learned in the curriculum around motivational interviewing and the health and society components regarding diet and physical exercise. I believe $\mathrm{KYHH}$ is a great opportunity not only to practice motivational interviewing, but also to reinforce knowledge of dietary and physical exercise guidelines in a real-life setting."

"[NUFSM] really stresses motivational interviewing and how important it is to get to know a patient. Yet there always isn't time to practice it much. That's where KYHH has been really helpful."

"I didn't know what motivational interviewing was before I started. I thought it was just giving people suggestions, but I learned through $\mathrm{KYHH}$ that it is more about empowering others to come up with their own plans for change."

"Ultimately KYHH will make me a better communicator and ultimately better doctor ... KYHH has made a difference in the way that I am able to interact with patients."

"We've had a very large experience with developing our communication skills, how to work with communities, how to empower them, how to develop sustainable programs, how to engage in conversation and work collaboratively."

"Participating in KYHH really allows you to keep growing even when you've been introduced to a lot of concepts already. As a second year I know what Motivational Interviewing is and I know the basic tenets of it, but now I am allowed to continue practicing and growing. Every patient experience that I have is different and I'm better at it. I learn from every patient in a different way."

"KYHH represents experiential learning in its purest form as we take what we learn in the classroom and see its impact in a very real setting."

Abbreviations: KYHH, Keep Your Heart Healthy; MSI, first-year medical student; MS2, second-year medical student; MS4, fourth-year medical student; NUFSM, Northwestern University Feinberg School of Medicine; OSCE, Objective Structured Clinical Examination.

progress, and serve as an assessment tool for student learning and growth through medical school. ${ }^{27}$ The eight competency objectives at NUFSM are: Patient-centered Medical Care, Effective Communication and Interpersonal Skills, Medical Knowledge and Scholarship, System Awareness and Team-based Care, Personal Awareness and Self-care, Community Engagement and Service, Continuous Learning and Quality Improvement, and Professional Behavior and Moral Reasoning.

These competencies, which are based on the educational competencies defined by the ACGME, serve as a launching point for all Northwestern medical school efforts. KYHH effectively addresses several of the competency points:

- Patient-centered Medical Care through participant CVD risk counseling and the provision of targeted recommendations based on participant-specific risk factors and neighborhood-specific resources.
- Effective Communication and Interpersonal Skills through the use of motivational interviewing for effective participant counseling, interaction with attending physicians, and encounters with community participants at screening events.

- Medical Knowledge and Scholarship through didactic and applied training sessions covering CVD epidemiology, risk, prevention, and lifestyle contributions to overall health.

- System Awareness and Team-based Care through involvement in high disease risk, low-resource neighborhoods promoting an understanding of barriers to health care access.

- Community Engagement and Service by offering students the opportunity to interact with real patients in the community setting as a supplement to simulated interactions in the classroom setting. 
- Continuous Learning and Quality Improvement through participation in experiential learning and involvement in the vertical learning model (ie, attending physicians mentor senior and junior students, senior students teach and provide feedback to junior medical students).

In post-screening surveys and in-person interviews, students confirmed that KYHH helped address these competency goals. Empirical evidence demonstrates that community programs linked to course-based learning objectives, such as the one described here, have a greater impact on students than elective or voluntary experiences. ${ }^{28}$ Furthermore, evidence suggests that there is an educational benefit for medical students who participate in service-learning outside of structured clinical settings in comparison to learning solely in clinical settings. ${ }^{28}$ Thus, KYHH has the potential to enhance medical school education.

\section{Limitations}

Though the KYHH pilot has generated positive feedback and has been successfully implemented as a service-learning opportunity for medical students, several limitations exist. Objective outcomes data are pending for this program. We did not compare those students involved in KYHH and those uninvolved, thus we are unable to determine empirically whether participation in KYHH improves students' performance on objective examinations, class rank, preclinical and clinical grades, or election to honor societies. Finally, KYHH students were recruited based on interest, creating a potential for volunteer bias in outcomes analysis.

\section{Future directions}

With support from students and faculty, the NUFSM Curriculum Committee approved $\mathrm{KYHH}$ as an elective course beginning September 2015 for the incoming class. To obtain elective credit, we will include a few changes including formalized student performance evaluations by attending physicians at community events, and a minimum attendance at ten community events. As with any curricular program, resources including staff and faculty involvement, supplies, and transportation have been required for KYHH to operate. The GE Foundation largely provided the monetary support for the pilot, and as the program has grown, additional resources have been provided by NUFSM. Furthermore, with the expansion of KYHH to up to 16 communities by 2016 , NUFSM will partner with additional community-based organizations and widen the students' ability to identify how different environmental, socioeconomic, and cultural dynamic factors affect the health of individuals and communities across Chicago.

Further analysis is needed to determine how participation in KYHH influences student education. Going forward, we plan to measure the effect of KYHH on academic outcomes such as performance on Objective Structured Clinical Examinations, clinical clerkships, and national medical licensing examinations. Preliminary results from community screenings have already been published. ${ }^{17}$ We anticipate that the effect of our program on community residents' health will continually be evaluated as the project moves forward.

\section{Conclusion}

In summary, KYHH has enhanced medical school education for participating NUFSM students by addressing several of the NUFSM and ACGME competencies. Through its novel collaboratively developed community-based screening and counseling model, KYHH may enhance medical students' ability to emphasize and counsel for prevention in their future practice. By integrating the program into the NUFSM curriculum, we hope that KYHH will continue to strengthen the education of medical students.

\section{Acknowledgments}

The authors thank the CDPH, Greater Humboldt Park Community Diabetes Empowerment Center, Family Focus Lawndale, Omron Corporation, American Heart Association Chicago Metro Affiliate, the KYHH Executive Committee, and SAC, faculty, and student volunteers for screening events, and all community resident participants. Funding for KYHH was provided by a grant from the GE Foundation with support from NUFSM.

\section{Disclosure}

The authors report no conflicts of interest in this work.

\section{References}

1. Centers for Disease Control and Prevention. Leading Causes of Death. Atlanta, GA: CDC; 2013. Available from: http://www.cdc.gov/nchs/ fastats/lcod.htm. Accessed May 24, 2014

2. NHLBI. Fact Book Fiscal Year 2012: Disease Statistics. Bethesda, MD: NHLBI; 2013.

3. CDPH. Healthy Chicago: A Public Health Agenda. Chicago, IL: Chicago Department of Public Health; 2011

4. Gorrindo P, Peltz A, Ladner TR, Miller BM, Robert F, Fowler J. Medical students as health educators at a student-run free clinic: improving the clinical outcomes of diabetic patients. Acad Med. 2014;89(4): 625-631

5. Burrows MS, Chauvin S, Lazarus CJ, Chehardy P. Required service learning for medical students: program description and student response. Teach Learn Med. 1999;11(4):223-231. 
6. Brush DR, Markert RJ, Lazarus CJ. The relationship between service learning and medical student academic and professional outcomes. Teach Learn Med. 2006;18(1):9-13.

7. Landy DC, Gorin MA, O'Connell MT. Student-led rural health fairs: attempting to improve medical education and access to health care. South Med J. 2011;104(8):598-603.

8. Balcazar H, Alvarado M, Ortiz G. Salud Para Su Corazon (health for your heart) community health worker model: community and clinical approaches for addressing cardiovascular disease risk reduction in Hispanics/Latinos. J Ambul Care Manage. 2011;34(4):362-372.

9. Balcazar H, Rosenthal EL, Brownstein JN, Rush CH, Matos S, Hernandez L. Community health workers can be a public health force for change in the United States: three actions for a new paradigm. Am J Public Health. 2011;101(12):2199-2203.

10. De las Nueces D, Hacker K, DiGirolamo A, Hicks LS. A systematic review of community-based participatory research to enhance clinical trials in racial and ethnic minority groups. Health Serv Res. 2012; 47(3 Pt 2):1363-1386.

11. Dodani S. Community-based participatory research approaches for hypertension control and prevention in churches. Int $J$ Hypertens. 2011;2011:273120.

12. Pennant M, Davenport C, Bayliss S, Greenheld W, Marshall T, Hyde C. Community programs for the prevention of cardiovascular disease: a systematic review. Am J Epidemiol. 2010;172(5):501-516.

13. Fancher TL, Keenan C, Meltvedt C, et al. An academic-community partnership to improve care for the underserved. Acad Med. 2011;86(2): 252-258.

14. Chastonay P, Zesiger V, Klohn A, et al. Development and evaluation of a community immersion program during preclinical medical studies: a 15-year experience at the University of Geneva Medical School. Adv Med Educ Pract. 2013;4:69-76.

15. Dent MM, Mathis MW, Outland M, Thomas M, Industrious D. Chronic disease management: teaching medical students to incorporate community. Fam Med. 2010;42(10):736-740.

16. AHA. My Life Check - Life's Simple 7. Available from: http:// mylifecheck.heart.org/. Accessed May 21, 2014.

17. Ducharme-Smith A, Klein DA, Rassiwala J, et al. The keep your heart healthy project: engaging medical students to reduce risk of cardiovascular disease. Jacobs J Epidemiol Prev. 2015;1(1):1-7.
18. LCME. IS-14-A: Service Learning. Available from: http://www.lcme. org/connections/connections_2013-2014/IS-14-A_2013-2014.htm. Accessed May 18, 2014.

19. Maurana CA, Goldenberg K. A successful academic-community partnership to improve the public's health. Acad Med. 1996;71(5):425-431.

20. Cauley K, Maurana CA, Clark MA. Service-learning for health professions students in the community: matching enthusiasm, talent and time with experience, real needs and schedules. In: Taylor B, editor. Expanding Boundaries: Serving and Learning. Washington, DC: Corporation for National Service; 1996;1:54-57.

21. Connors K, Seifer SD. Overcoming a century of town-gown relations: redefining relationships between communities and academic health centers through community-campus partnerships. In: Taylor B, editor. Expanding Boundaries: Serving and Learning. Washington, DC: Corporation for National Service; 1997;2:2-8.

22. Dornan T, Littlewood S, Margolis SA, Scherpbier A, Spencer J, Ypinazar V. How can experience in clinical and community settings contribute to early medical education? A BEME systematic review. Med Teach. 2006;28(1):3-18.

23. Hunt JB, Bonham C, Jones L. Understanding the goals of service learning and community-based medical education: a systematic review. Acad Med. 2011;86(2):246-251.

24. Furze J, Black L, Peck K, Jensen GM. Student perceptions of a community engagement experience: exploration of reflections on social responsibility and professional formation. Physiother Theory Pract. 2011;27(6):411-421.

25. Averill NJ, Sallee JM, Robinson JT, et al. A first-year community-based service learning elective: design, implementation, and reflection. Teach Learn Med. 2007;19(1):47-54.

26. Olney CA, Livingston JE, Fisch S, Talamantes MA. Becoming better health care providers: outcomes of a primary care service-learning project in medical school. J Prev Interv Community. 2006;32(1-2):133-147.

27. NUFSM. Competency Compass. Available from: http://www.feinberg. northwestern.edu/Education/curriculum/principles/competencies/index. html. Accessed October 24, 2014

28. Driscoll A, Holland B, Gelmon S, Kerrigan S. A case study assessment model for evaluating the impact of service-learning on students, faculty, institutions and communities. Mich J Community Serv Learn. 1996;3: $22-31$. 


\section{Supplementary material}

Development and evaluation of a service-learning model for preclinical student education in cardiovascular disease prevention.

\section{Keep your heart healthy!}

We want to help you learn more about keeping your heart healthy. There will be 3 steps:

1) Assess: answer some questions and we will check your blood pressure \& body weight

2) Discuss: we will talk to you about the things you can do to avoid a heart attack or stroke

3) Act: we will help you set goals for healthy living and connect you with resources to help you

Please answer the questions below. This will help us to discuss your heart health

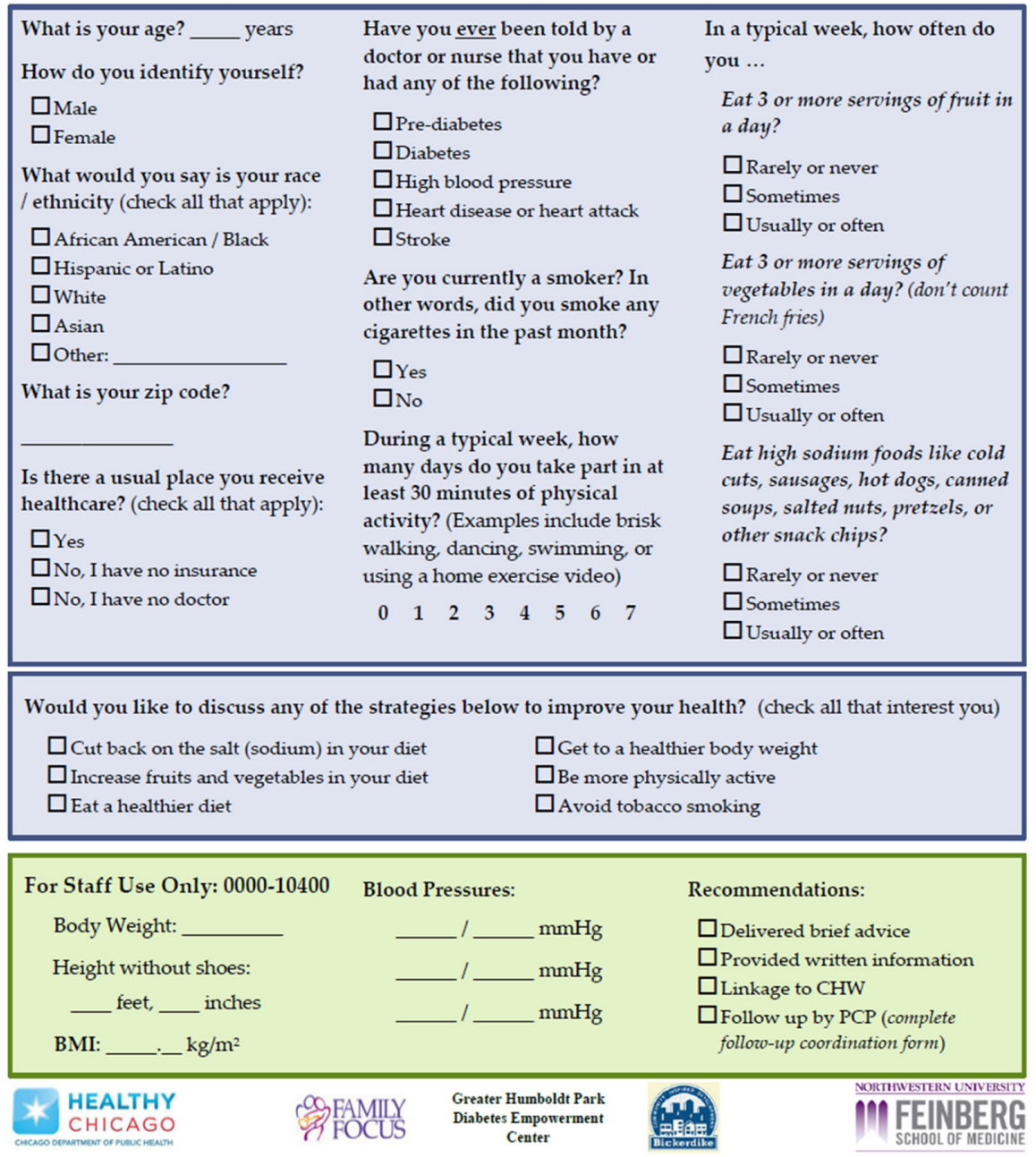

Figure SI English language screening and counseling questionnaire.

Advances in Medical Education and Practice

\section{Publish your work in this journal}

Advances in Medical Education and Practice is an international, peerreviewed, open access journal that aims to present and publish research on Medical Education covering medical, dental, nursing and allied health care professional education. The journal covers undergraduate education, postgraduate training and continuing medical education including emerging trends and innovative models linking education, research, and health care services. The manuscript management system is completely online and includes a very quick and fair peer-review system. Visit http://www.dovepress.com/testimonials.php to read real quotes from published authors.

Submit your manuscript here: http://www.dovepress.com/advances-in-medical-education-and-practice-journal 